\title{
Modeling of metastable argon atoms in a direct-current glow discharge
}

\author{
A. Bogaerts* and R. Gijbels \\ Department of Chemistry, University of Antwerp (UIA), Universiteitsplein 1, B-2610 Wilrijk-Antwerp, Belgium
}

(Received 17 February 1995; revised manuscript received 8 May 1995)

\begin{abstract}
To calculate the behavior of metastable argon atoms in a direct-current glow discharge, a balance equation is constructed, taking into account all known production and loss processes of the metastable atoms. Density profiles and fluxes of the metastable atoms are computed. The relative importance of different production and loss processes determining the metastable density is calculated for the case of a molybdenum cathode in pure argon. Besides electron-impact excitation, fast-ion and atom-impact excitation are found to be the dominant production processes at the high voltages used here, while loss of the metastable atoms is caused predominantly by diffusion and also by electron quenching to the nearby resonant states. The role of metastable atoms in the total discharge is investigated. They are found to play a minor role in the ionization of argon atoms, but their part in the ionization of sputtered molybdenum cathode atoms appears to be rather important. Moreover, they seem to have a significant effect on the secondary electron emission at the cathode. The investigation also includes the influence of pressure, voltage, and current on the metastable densities and fluxes, on the relative importance of the different production and loss processes, and on the role of metastable atoms in the entire discharge.
\end{abstract}

PACS number(s): $34.50 . \mathrm{Fa}$

\section{INTRODUCTION}

Glow discharges are applied in the microelectronics industry as plasma processing devices for ion etching, thin-film deposition, and plasma treating of surfaces [1]. Moreover, they are gaining increasing interest as atomizationexcitation-ionization sources for analytical spectrometry $[2,3]$. To improve the results in these application fields, thorough insight into glow discharge is necessary. This can be acquired by mathematical modeling. In this work, an investigation is made concerning the role of metastable argon atoms in the discharge. This is important from an analytical point of view, since it is suggested [4-6] that the ionization of sputtered (analytically important) atoms by metastable argon atoms (so-called Penning ionization) is one of the dominant ionization mechanisms.

Metastable atoms have been studied by several authors both experimentally and theoretically. Metastable densities can experimentally be measured by optical absorption techniques. In theoretical work, a balance equation including different production and loss terms is constructed to calculate the metastable densities. Experimental measurements were performed, for example, for $\mathrm{He}$ as a function of discharge conditions in Ref. [7], for Ar in a microwave boosted glow discharge in Ref. [8], and for $\mathrm{Ne}$ in an rf glow discharge in Ref. [6]. In Ref. [4] only relative absorption signals of $\mathrm{Ar}$ metastable atoms as a function of current and pressure were recorded. In Refs. [9] and [10] Ar metastable densities were measured in a Grimm-type glow discharge and in the afterglow of a pulsed discharge, respectively, and some mechanisms for the formation of Ar metastable atoms were suggested. Ar metastable densities have also been measured in Refs. [11] and [12]. In the latter work, a comparison was made with Ar metastable densities calculated from a

\footnotetext{
*Author to whom correspondence should be addressed.
}

coupled-electron-metastable-atom model. A comparison between experimental and calculated metastable densities was also carried out in Ref. [13] for $\mathrm{He}$ in a dc and rf glow discharge, in Ref. [14] for $\mathrm{He}, \mathrm{Ne}$, and Ar, and in Refs. [15] and [16] for He. In Ref. [17] a combined fluid model was developed for the electrons, argon ions, and argon metastable atoms in order to investigate the effect of metastable atoms in the discharge. In Refs. [18-23] rate constants of a number of collision processes responsible for the destruction of metastable atoms were obtained by combining balance equations with the measured time-dependent variation of the metastable densities or by analyzing the dependence of the decay constants upon pressure.

The theoretical models mentioned here take into account a number of production and loss processes-one model is more complete than the other-but none of these models considers Penning ionization of sputtered atoms as a possible loss mechanism and fast-argon-ion and atom-impact excitation as possible production mechanisms for the argon metastable atoms. Of course, these models are meant to describe glow discharges for etching and deposition, which operate at lower voltages at which the above-mentioned processes may not be important. However, in glow discharges used as an ion source for mass spectrometry, like we try to describe, the operating voltages are much higher and those processes cannot be neglected. In this work, the Ar metastable density is calculated in a dc glow discharge, with a balance equation taking into account all known production and loss mechanisms. The calculated metastable densities and fluxes throughout the discharge are compared with previously calculated ion and sputtered atom densities and fluxes. Moreover, a comparison is made with metastable densities reported in the literature. The relative importance of the various production and loss processes determining the metastable density is discussed. The study also addresses the relative contribution of metastable atoms in the ionization of argon atoms and sputtered atoms and in the secondary elec- 
tron emission from the cathode. Finally, the influence of pressure, voltage, and current on the metastable densities and fluxes, on the relative importance of different production and loss processes, and on the relative role of metastable atoms in the discharge is investigated.

\section{DESCRIPTION OF THE MODEL}

The model we developed for the metastable atoms is one dimensional, i.e., it applies to a discharge between two infinitely wide electrodes (anode and cathode) so that quantities vary only with distance from the electrodes. Besides the metastable argon atoms, the plasma is assumed to consist of neutral ground-state argon atoms at rest, uniformly distributed throughout the discharge, fast and slow electrons and argon ions, described in a hybrid model [24], fast argon atoms, and also sputtered atoms. The density of the latter is calculated by a simple one-dimensional diffusion equation $[25,26]$

$$
D \frac{d^{2} n}{d x^{2}}=-J_{0} F_{T}(x)
$$

where $J_{0}$ is the flux of sputtered atoms, calculated from an empirical formula of the sputtering yield [27] and the flux energy distribution of argon ions and fast argon atoms bombarding the cathode [28], and $F_{T}(x)$ is the thermalization profile of sputtered atoms, i.e., the distribution of sputtered atoms after they have been thermalized in the plasma [29]. This is only an approximate formula, since ionization of Mo and self-sputtering of the cathode by Mo ions have not yet been considered here. However, both effects are opposed to each other and the overall effect is rather small. Moreover, this approximation will not affect the metastable argon density since the sputtered atoms play only a minor role in determining the metastable density (see later).

Argon atoms possess two metastable levels, lying closely to each other, i.e., the $\left(3 p^{5} 4 s\right){ }^{3} P_{2}$ level and the $\left(3 p^{5} 4 s\right)^{3} P_{0}$ level, at 11.55 and $11.72 \mathrm{eV}$ above the ground state, respectively. The ${ }^{3} P_{0}$ level is stated to be populated by a fraction of only $10-20 \%$ of the ${ }^{3} P_{2}$ level [22,23]. Since for our purpose only the total metastable density is important, we have combined the two metastable levels in one collective level lying at $11.55 \mathrm{eV}$. Also two resonant levels are lying closely to the metastable levels, i.e., the $\left(3 p^{5} 4 s\right)^{3} P_{1}$ level and the $\left(3 p^{5} 4 s\right)^{1} P_{1}$ level, at 11.62 and $11.83 \mathrm{eV}$ above the ground state, respectively. These levels can decay to the ground state by emission of radiation.

The density of metastable atoms is calculated by solving a balance equation considering different production and loss processes. The production processes incorporated in the model are (i) fast-electron-impact excitation from groundstate argon atoms, including cascading from higher-energy levels, (ii) fast-ion and fast-atom-impact excitation from ground-state argon atoms, and (iii) radiative recombination between $\mathrm{Ar}^{+}$ions and slow electrons. Dissociative recombination between $\mathrm{Ar}_{2}^{+}$and electrons is neglected since $\mathrm{Ar}_{2}^{+}$is assumed not to be present in the plasma.

Loss processes taken into account comprise (i) fastelectron-impact ionization from the metastable level; (ii) fast-electron-impact excitation from the metastable level to higher-energy levels; (iii) transfer to the nearby resonant states by collision with thermalized electrons, which is called electron quenching; (iv) metastable-atom-metastable-atom collisions, resulting in ionization of one of the atoms; (v) Penning ionization of sputtered cathode atoms; (vi) two-body collisions with ground-state argon atoms [i.e., (a) collisional transfer from the metastable states to nearby resonant levels and (b) collision-induced emission); and (vii) three-body collisions with ground-state argon atoms, resulting in the formation of $\mathrm{Ar}_{2}^{*}$. These different production and loss processes are summarized in Table I. An additional loss process is diffusion to the walls followed by deexcitation at the walls. We assume the gas to be pure argon, so that destruction of argon metastable atoms due to reactions with impurity gas molecules (quenching) can be neglected. These production and loss processes result in the balance equation

$$
\frac{\partial n_{\mathrm{Ar}_{m}^{*}}}{\partial t}-D \underset{\mathrm{Ar} *}{*} \frac{\partial^{2} n_{\mathrm{Ar}_{m}^{*}}}{\partial x^{2}}=r_{\text {prod }}(x)-r_{\mathrm{loss}}(x),
$$

where

$$
\begin{aligned}
r_{\text {prod }}(x)= & r_{e, \text { exc }}(x)+r_{i / a, \text { exc }}(x)+k_{\text {recom }} n_{e_{s}}(x) n_{\mathrm{Ar}^{+}}(x), \\
r_{\text {loss }}(x)= & r_{\text {ion,met }}(x)+r_{\text {exc,met }}(x)+k_{\text {quen }} n_{e_{s}}(x) n_{\mathrm{Ar}_{m}^{*}}(x) \\
& +2 k_{\text {met }}\left[n_{\mathrm{Ar}_{m}^{*}}(x)\right]^{2}+k_{\mathrm{PI}} n_{M}(x) n_{\mathrm{Ar}_{m}^{*}}(x) \\
& +k_{2 \mathrm{~B}^{n}} n_{\mathrm{Ar}^{0}} n_{\mathrm{Ar}_{m}^{*}}(x)+k_{3 \mathrm{~B}}\left(n_{\mathrm{Ar}_{0}}\right)^{2} n_{\mathrm{Ar}_{m}^{*}}(x) .
\end{aligned}
$$

The first term on the left-hand side in Eq. (2) is the timedependent variation, which becomes zero at steady state. The second term relates to diffusion; the diffusion coefficient $D_{\mathrm{Ar}_{m}^{*}}$ is taken to be $54 \mathrm{~cm}^{2} \mathrm{~s}^{-1}$ at 1 Torr [18]. The first term on the right-hand side in Eq. (2) includes the production terms. $r_{e, \text { exc }}(x)$ is the electron-impact excitation rate, which is calculated from an electron Monte Carlo simulation. The cross section for this process as a function of the electron energy is taken from Ref. [30]. $r_{i / a, \operatorname{exc}}(x)$ is the ion- and atom-impact excitation rate, which is calculated from an ion and atom Monte Carlo simulation [28,31] with the cross section as a function of the ion and atom energy taken from Ref. [32]. $k_{\text {recom }}$ is the rate constant of electron-ion radiative recombination, which is taken to be $10^{-11} \mathrm{~cm}^{3} \mathrm{~s}^{-1}$ [33]. The second term on the right-hand side in Eq. (2) comprises all the loss terms. $r_{\text {ion,met }}(x)$ and $r_{\text {exc,met }}(x)$ are the electronimpact ionization and excitation rates from the metastable level, respectively; they are calculated in an electron Monte Carlo model with the metastable density known from a previous solution of the metastable balance equation. Cross sections of these processes as a function of the electron energy are adopted from Ref. [34] for electron-impact ionization and from Ref. [35] for electron-impact excitation. $k_{\text {quen }}$ is the rate constant of electron quenching to the nearby resonant states, which is taken to be $2 \times 10^{-7} \mathrm{~cm}^{3} \mathrm{~s}^{-1}$ [17]. $k_{\text {met }}$ represents the rate constant of metastable-atom-metastable-atom collisions, which is assumed to have a value of $6.4 \times 10^{-10}$ $\mathrm{cm}^{3} \mathrm{~s}^{-1}[11,12] . k_{\mathrm{PI}}$ is the rate constant of Penning ionization of sputtered atoms. Reliable values for the cross sections of this process between argon metastable atoms and metals are very difficult to find. We used an empirical formula [36], 
TABLE I. Production and loss processes of metastable argon atoms incorporated in the model. $m, r$, and $*$ refer to a metastable state, a resonant state, and an arbitrary excited state, respectively.

\begin{tabular}{|c|c|c|}
\hline Production processes & & Rate \\
\hline $\begin{array}{l}\text { (i) } \mathrm{Ar}^{0}+e_{f}^{-} \rightarrow \mathrm{Ar}_{m}^{*}+e_{f}^{-} \\
\text {(ii) } \mathrm{Ar}^{0}+\operatorname{Ar}_{f}^{+}\left(\operatorname{Ar}_{f}^{0}\right) \rightarrow \mathrm{Ar}_{m}^{*}+\operatorname{Ar}_{f}^{+}\left(\operatorname{Ar}_{f}^{0}\right) \\
\text { (iii) } \mathrm{Ar}^{+}+e_{s}^{-} \rightarrow \mathrm{Ar}_{m}^{*}+h \nu\end{array}$ & $\begin{array}{l}\text { electron-impact excitation } \\
\text { ion- (atom-) impact excitation } \\
\text { electron-ion radiative recombination }\end{array}$ & $\begin{array}{c}\sigma_{e, \mathrm{exc}}(E)^{\mathrm{a}} \\
\sigma_{i / a, \mathrm{exc}}(E)^{\mathrm{b}} \\
k_{\text {recom }}=10^{-11} \mathrm{~cm}^{3} \mathrm{~s}^{-1 \mathrm{c}}\end{array}$ \\
\hline Loss processes & & Rate \\
\hline $\begin{array}{l}\text { (i) } \mathrm{Ar}_{m}^{*}+e_{f}^{-} \rightarrow \mathrm{Ar}^{+}+2 e^{-} \\
\text {(ii) } \mathrm{Ar}_{m}^{*}+e_{f}^{-} \rightarrow \mathrm{Ar}^{*}+e^{-} \\
\text {(iii) } \mathrm{Ar}_{m}^{*}+e_{s}^{-} \rightarrow \mathrm{Ar}_{r}^{*}+e_{s}^{-} \\
\text {(iv) } \mathrm{Ar}_{m}^{*}+\mathrm{Ar}_{m}^{*} \rightarrow \mathrm{Ar}^{0}+\mathrm{Ar}^{+}+e^{-} \\
\text {(v) } \mathrm{Ar}_{m}^{*}+M^{0} \rightarrow \mathrm{Ar}^{0}+M^{+\left(^{*}\right)}+e^{-} \\
\text {(vi) } \mathrm{Ar}_{m}^{*}+\mathrm{Ar}^{0} \rightarrow \mathrm{Ar}^{0}+\mathrm{Ar}^{0} \\
\text { (vii) } \mathrm{Ar}_{m}^{*}+2 \mathrm{Ar}^{0} \rightarrow \mathrm{Ar}_{2}^{*}+\mathrm{Ar}^{0} \\
\text { (viii) diffusion }\end{array}$ & $\begin{array}{l}\text { electron-impact ionization } \\
\text { electron-impact excitation } \\
\text { electron quenching } \\
\text { metastable-metastable collision } \\
\text { Penning ionization } \\
\text { two-body collision } \\
\text { three-body collision }\end{array}$ & $\begin{array}{c}\sigma_{\text {ion,met }}(E)^{\mathrm{d}} \\
\sigma_{\text {exc,met }}(E)^{\mathrm{e}} \\
k_{\text {quen }}=2 \times 10^{-7} \mathrm{~cm}^{3} \mathrm{~s}^{-1 \mathrm{f}} \\
k_{\mathrm{met}}=6.4 \times 10^{-10} \mathrm{~cm}^{3} \mathrm{~s}^{-1 \mathrm{~g}} \\
k_{\mathrm{PI}}=2.6 \times 10^{-10} \mathrm{~cm}^{3} \mathrm{~s}^{-1 \mathrm{~h}} \\
k_{2 \mathrm{~B}}=2.3 \times 10^{-15} \mathrm{~cm}^{3} \mathrm{~s}^{-1 \mathrm{i}} \\
k_{3 \mathrm{~B}}=1.4 \times 10^{-32} \mathrm{~cm}^{6} \mathrm{~s}^{-1 \mathrm{i}} \\
D_{\mathrm{Ar}_{m}^{*}}=54 \mathrm{~cm}^{2} \mathrm{~s}^{-1 \mathrm{j}}\end{array}$ \\
\hline
\end{tabular}

${ }^{\mathrm{a} R e f e r e n c e ~[30] .}$

${ }^{\mathrm{b}}$ Reference [32].

${ }^{\mathrm{c}}$ Reference [33].

${ }^{\mathrm{d}}$ Reference [34].

${ }^{\mathrm{e}}$ Reference [35].

which we fitted to some experimentally obtained cross sections $[36,37]$ in order to arrive at approximate values for other elements. The Penning ionization cross section of Mo at thermal energies was in this way computed to be $5.8 \times 10^{-15} \mathrm{~cm}^{2}$, which corresponds to a $k_{\mathrm{PI}}$ of $2.6 \times 10^{-10}$ $\mathrm{cm}^{3} \mathrm{~s}^{-1} \cdot k_{2 \mathrm{~B}}$ and $k_{3 \mathrm{~B}}$ are the rate constants of two-body and three-body collisions with argon ground-state atoms, respectively. Their numerical values are taken from Ref. [22], i.e., $k_{2 \mathrm{~B}}=2.3 \times 10^{-15} \mathrm{~cm}^{3} \mathrm{~s}^{-1}$ and $k_{3 \mathrm{~B}}=1.4 \times 10^{-32} \mathrm{~cm}^{6} \mathrm{~s}^{-1}$. Fi-

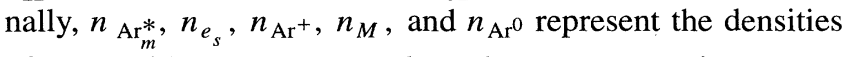
of metastable argon atoms, slow electrons, argon ions, sputtered atoms, and argon ground-state atoms, respectively. The loss due to diffusion and subsequent deexcitation at the walls is determined by the boundary conditions, i.e., $n_{\mathrm{Ar}_{m}^{*}}=0$ at $x=0$ and $L$. The balance equation [Eq. (2)] is discretized and then solved with the Thomas algorithm [38].

The metastable model forms a part of a big model, consisting of different "sub-models" for different plasma species. A Monte Carlo model describes the fast electrons, taking into account electron-impact excitation, ionization, and elastic collisions. A fluid model handles the slow electrons and argon ions and is combined with the Monte Carlo electron model to form a hybrid model [24]. The argon ions and the fast argon atoms are treated with a Monte Carlo model in the cathode dark space (CDS) only [28,31]. Collision processes incorporated in that model comprise charge transfer (for the ions) and elastic collisions and ion- and atom-impact ionization and excitation (for the ions and fast atoms).

The metastable model is computed iteratively with the hybrid Monte Carlo fluid model [24]. The metastable model affects the hybrid model in the following ways: (i) the metastable density determines the electron-impact excitation and ionization rates from the metastable levels, (ii) the metastable flux at the cathode plays a role in secondary electron

${ }^{\mathrm{f}}$ Reference [17].
${ }_{\mathrm{g}}$ Reference [11,12].
${ }^{\mathrm{h}}$ Reference [36,37].
${ }^{\mathrm{i}}$ Reference [22].
${ }^{\mathrm{j}}$ Reference [18].

emission, and (iii) some of the loss processes (i.e., metastable-atom-metastable-atom collisions and electronimpact ionization from the metastable levels) cause the creation of new electrons and argon ions, whereas (iv) the production process of electron-ion recombination can in principle decrease the number of ions and electrons, although this process will be negligible. The iterative procedure is explained in Fig. 1, i.e., the electron Monte Carlo part of the fluid model calculates the relevant electron-impact excitation and ionization rates that are used as input in the metastable model. The results of the metastable model, i.e., the metastable density and flux, as well as the effects on the ion and electron densities are inserted in the hybrid model, etc. This procedure is repeated until convergence is reached (typically after 3-4 iterations).

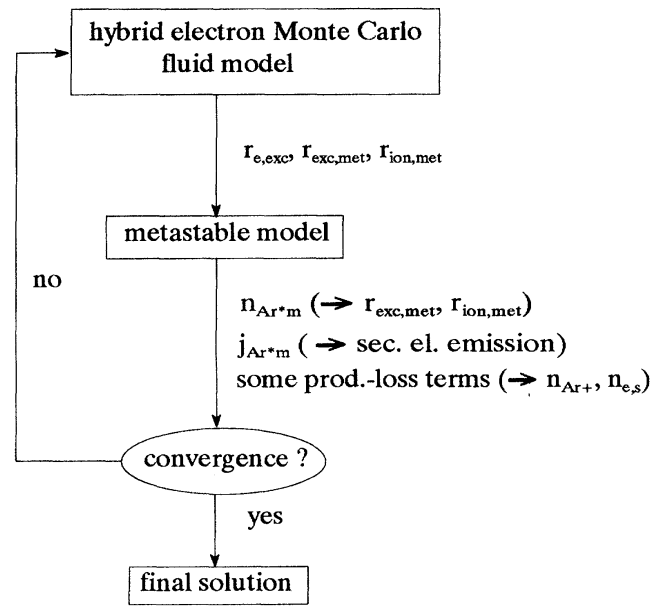

FIG. 1. Flowchart of the model. 


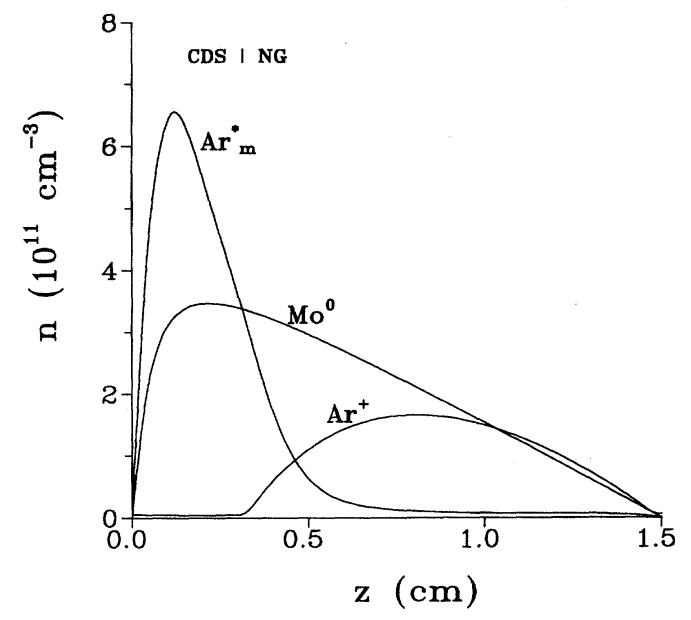

FIG. 2. Density profiles of Ar metastable atoms, Ar ions, and sputtered Mo atoms as a function of distance from the cathode (Mo in Ar, $850 \mathrm{~V}, 75 \mathrm{~Pa}$ ). The boundary between cathode dark space and negative glow is at $0.3 \mathrm{~cm}$ from the cathode.

\section{RESULTS AND DISCUSSION}

Calculations were carried out for a molybdenum cathode in an argon discharge at $75 \mathrm{~Pa}$ gas pressure and $850 \mathrm{~V}$ discharge voltage. The discharge cell is assumed to be $1.5 \mathrm{~cm}$ long and infinitely wide. Figure 2 shows the density profile of the metastable Ar atoms together with the Ar ion density and the density of the sputtered Mo atoms. The Ar metastable density is zero at the walls due to wall deexcitation and reaches a maximum of about $6.5 \times 10^{11} \mathrm{~cm}^{-3}$ at $0.12 \mathrm{~cm}$ from the cathode, whereafter it decreases rapidly. We compared this result with calculated or measured values found in the literature $[8,9,11,14,21]$. Depending on discharge conditions, all these values vary between $2 \times 10^{10}$ and $5 \times 10^{13}$ $\mathrm{cm}^{-3}$. Hence our calculated values certainly lie in the correct range of order of magnitude.

Figure 2 also shows that the Ar metastable density is of the same order of magnitude as the Ar ion density and the sputtered Mo atom density at these discharge conditions. The relative magnitude of these densities varies somewhat with discharge conditions, i.e., the argon ion density and especially the sputtered atom density increase more rapidly with voltage and pressure than the metastable density, but they always remain in the same order of magnitude. This is also experimentally found by Ferreira, Loureiro, and Ricard [12] and it is also stated in general terms in Ref. [39].

The flux of the Ar metastable atoms goes through zero at the position of maximum Ar metastable density. To the left of this maximum it is negative (directed towards the cathode) and to the right it is positive (directed towards the anode). The flux of metastable atoms bombarding the cathode is calculated to be $1.5 \times 10^{15} \mathrm{~cm}^{-2} \mathrm{~s}^{-1}$ at these discharge conditions. Comparing this with the argon ion flux bombarding the cathode $\left(j_{\mathrm{Ar}^{+}}\right.$is calculated to be $\left.4.74 \times 10^{15} \mathrm{~cm}^{-2} \mathrm{~s}^{-1}\right)$, we conclude that about $24 \%$ of the flux bombarding the cathode is due to argon metastable atoms, whereas the argon ions account for about $76 \%$. Supposing that the secondary electron emission coefficient due to $\mathrm{Ar}$ ion and $\mathrm{Ar}$ metastable bombardment is comparable (i.e., approximately 0.12), this

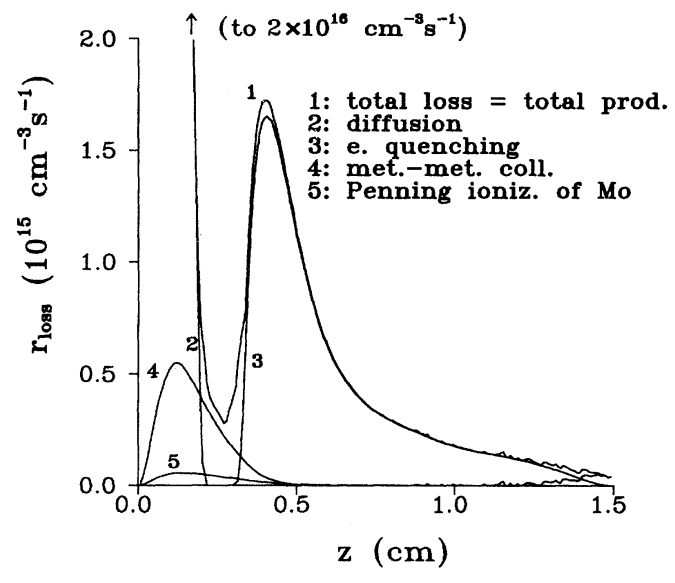

FIG. 3. Contribution of different loss processes determining the Ar metastable density, as a function of distance from the cathode (Mo in Ar, $850 \mathrm{~V}, 75 \mathrm{~Pa}$ ).

leads to a relative contribution of Ar metastable atom induced electron-impact emission at the cathode of about $24 \%$ at these discharge conditions.

In Fig. 3 the relative importance of different loss processes determining the Ar metastable density is illustrated. At steady state, the total loss rate (curve 1) is equal to the total production rate. This total production rate is mainly given by two processes, i.e., close to the cathode where the ions and atoms have high energies, it is almost completely determined by ion- and atom-impact excitation, which appears to be a very efficient process at high energies [32], leading to a very high production rate. Farther in the discharge, especially in the beginning of the negative glow, electron-impact excitation is the predominant production mechanism, leading to the second peak in the production rate at about $0.5 \mathrm{~cm}$. Integrated over the total discharge region, ion- and atom-impact excitation account for about $73.5 \%$ of the total production whereas electron-impact excitation is responsible for about $17 \%$. About $9.5 \%$ is ascribed to diffusion from regions with higher metastable density. Electron-ion recombination contributes only about $0.01 \%$ to the production rate. Concerning the loss of metastable atoms, two processes appear to be predominant. Near the cathode, the high production rate is mainly balanced by diffusion (curve 2), which tends to spread out the metastable density slightly farther in the discharge. In the negative glow, the loss is almost completely caused by electron quenching to the resonant states (curve 3). This process is negligible in the CDS since the thermalized electron density is nearly zero in that region [24]. Two other loss processes, however, seem to contribute in the CDS, i.e., metastable-metastable collisions (curve 4) and Penning ionization of sputtered Mo atoms (curve 5). Integrated over the total discharge, the loss due to diffusion amounts to about $71.6 \%$, electron quenching is responsible for about $21.9 \%$, and metastable-metastable collisions and Penning ionization amount to about $5.1 \%$ and $0.71 \%$, respectively. The other loss processes taken into account are still less important, i.e., electron-impact excitation and ionization from the metastable states contribute about $0.23 \%$ and $0.008 \%$ and the loss due to two-body and three-body collisions represents about $0.37 \%$ and $0.04 \%$. 


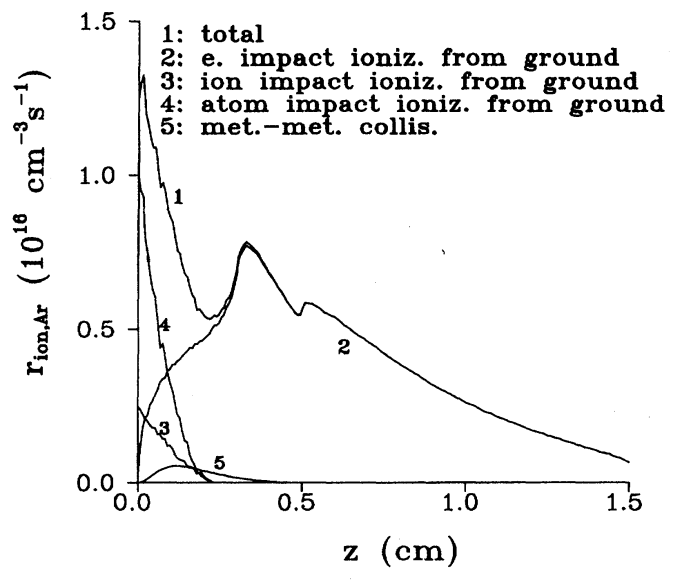

FIG. 4. Contribution of different ionization mechanisms to the ionization of $\mathrm{Ar}$ atoms, as a function of distance from the cathode (Mo in Ar, $850 \mathrm{~V}, 75 \mathrm{~Pa}$ ).

Two of these loss processes of the metastable atoms lead to the creation of $\mathrm{Ar}$ ions, i.e., electron-impact ionization from metastable levels and metastable-atom-metastableatom collisions. We compared these two processes with the direct electron-impact ionization of ground-state Ar atoms to investigate their relative contribution in the total ionization of Ar. In analogy with ion- and atom-impact excitation, also ion- and atom-impact ionization from the ground state are included. These processes appear indeed to be non-negligible at high voltages [31]. In Fig. 4 it is shown that the majority of Ar ions is formed by direct electron-impact ionization of ground-state atoms, although ion- and especially atomimpact ionization become increasingly important close to the cathode. The contribution of metastable-atom-metastableatom collisions is only small and that of electron-impact ionization from metastable levels is completely negligible at these discharge conditions. Integrated over the total discharge region, the relative contributions of electron-, ion-, and atom-impact ionization amount to about $82.5 \%, 3.8 \%$, and $12.0 \%$, respectively. Metastable-atom-metastable-atom collisions contribute about $1.72 \%$, whereas electron-impact ionization from the metastable levels amounts to about $0.003 \%$ only.

As said before, it is generally assumed that metastable atoms can play an important role in the ionization of sputtered atoms by Penning ionization. It is accepted that three processes come into play in the ionization of sputtered atoms: (i) electron-impact ionization, (ii) Penning ionization, and (iii) charge-transfer between Ar ions and sputtered atoms. The cross section of electron-impact ionization as a function of the electron energy can be found in Ref. [40]. The cross section for charge transfer between Ar ions and metals is even more difficult to find than the corresponding value for Penning ionization. Hence in order to find at least an approximated value for $\mathrm{Ar}^{+}$-Mo we used the following strategy. It is generally known [41-43] that the chargetransfer process is most likely when the energy difference between levels of the bombarding ion and the created ion is sufficiently small and that the probability of the charge transfer decreases rapidly with increasing energy difference. This type of collision can only be treated adequately using quan-

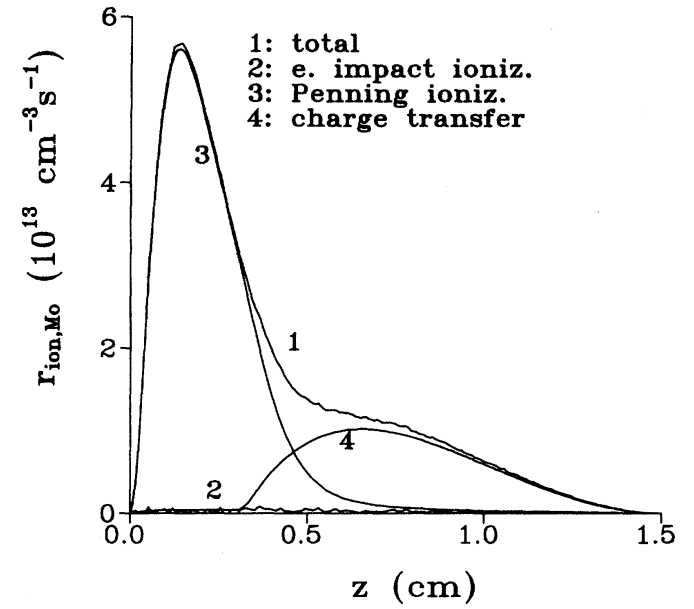

FIG. 5. Contribution of different ionization mechanisms to the ionization of Mo atoms, as a function of distance from the cathode (Mo in Ar, $850 \mathrm{~V}, 75 \mathrm{~Pa}$ ).

tum mechanics. In Refs. [42] and [43] cross sections of Penning ionization and charge transfer were measured for $\mathrm{He}^{+}-\mathrm{Cd}$ and $\mathrm{He}^{+}-\mathrm{Zn}$, respectively. In both cases we see that (i) the cross sections are almost identical to each other within a factor 1.5 and (ii) both $\mathrm{Cd}^{+}$and $\mathrm{Zn}^{+}$possess energy levels lying close to the $\mathrm{He}^{+}$ground-state level. Therefore we assume that if the energy difference between levels is small, the charge-transfer cross section is nearly equal to the Penning ionization cross section. Applying this to $\mathrm{Ar}^{+}-\mathrm{Mo}$ where the energy difference between excited Mo levels and the $\mathrm{Ar}^{+}$ground-state level was found to be small, we assume that the cross section of charge transfer for this system is equal to the Penning ionization cross section. This strategy is only a rough approximation, but since we did not find more accurate data, we will have to be satisfied with this solution, keeping in mind that the obtained results will be subject to considerable uncertainties. Also, the conclusions of these calculations are not necessarily true for other cathode materials.

We compared the relative contribution of electron-impact ionization, Penning ionization, and charge transfer to the ionization of sputtered Mo atoms, as illustrated in Fig. 5. Since the cross sections of Penning ionization and charge transfer are assumed equal to each other, the relative contribution of these two processes reflects the densities of Ar metastable atoms and Ar ions, respectively. In Fig. 2 it was shown that the Ar metastable density was higher than the Ar ion density, which results in a higher contribution of Penning ionization. The exact relative importance of these two processes has to be considered with caution due to the cross sections that are not exactly known, but it is obvious from Fig. 5 that both these processes are much more important for the ionization of Mo atoms than electron-impact ionization. Indeed, the cross section of electron-impact ionization is of the order of $10^{-16} \mathrm{~cm}^{2}$, which is considerably lower than the cross sections of Penning ionization and charge transfer (i.e., $5.8 \times 10^{-15} \mathrm{~cm}^{2}$ ) and the fast electron density is also clearly lower than the Ar ion and metastable densities [24]. Integrated over the total discharge region, the relative contribution of Penning ionization, charge transfer, and electronimpact ionization of Mo atoms comprise $69.9 \%, 28.9 \%$, and 

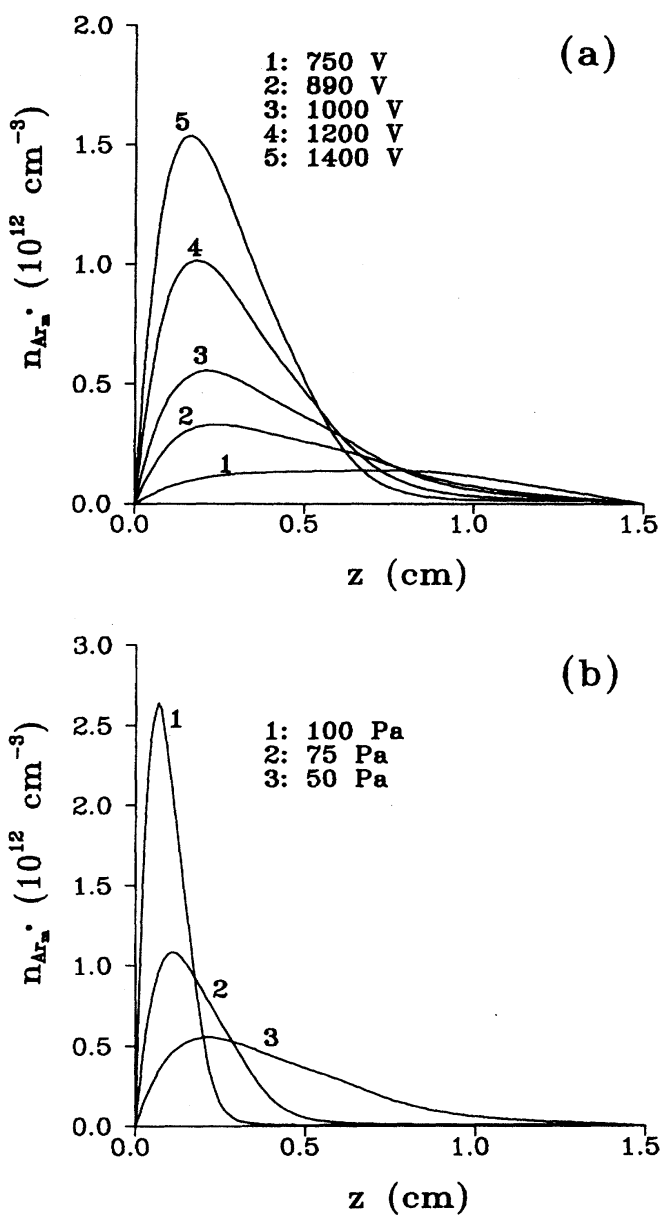

FIG. 6. Density profiles of Ar metastable atoms (a) as a function of voltage at $50 \mathrm{~Pa}$ and (b) as a function of pressure at $1000 \mathrm{~V}$ (Mo in $\mathrm{Ar}$ ).

$1.2 \%$, respectively. This result agrees with the statement in literature $[4-6,39]$ that Penning ionization is the dominant ionization process of sputtered atoms in an analytical glow discharge. For example, in Ref. [4] it is reported that the relative contribution of Penning ionization lies between $40 \%$ and $80 \%$, which is in agreement with our results. Concerning charge transfer, the findings differ: in Refs. [4,6,39] charge transfer is not even mentioned as ionization mechanism, whereas Steers and co-workers $[41,44]$ demonstrated that charge transfer is very important for $\mathrm{Ar}^{+}-\mathrm{Cu}$, which is also a system with a small energy difference between levels. In Ref. [5] it was suggested that charge transfer is negligible at low pressures and currents (0.3-2 Torr, 1-5 mA) but becomes increasingly important at higher pressures and currents.

Comparing Figs. 4 and 5, we see that the total ionization rate of $\mathrm{Ar}$ atoms is about 200 times higher than the total ionization rate of Mo atoms, although the overall density of Ar atoms (approximately $10^{16} \mathrm{~cm}^{-3}$ ) is about four orders of magnitude higher than the sputtered Mo atom density. This means that the ionization of sputtered atoms is much more efficient than the ionization of Ar, which is also generally known from mass spectrometric measurements. Hence the

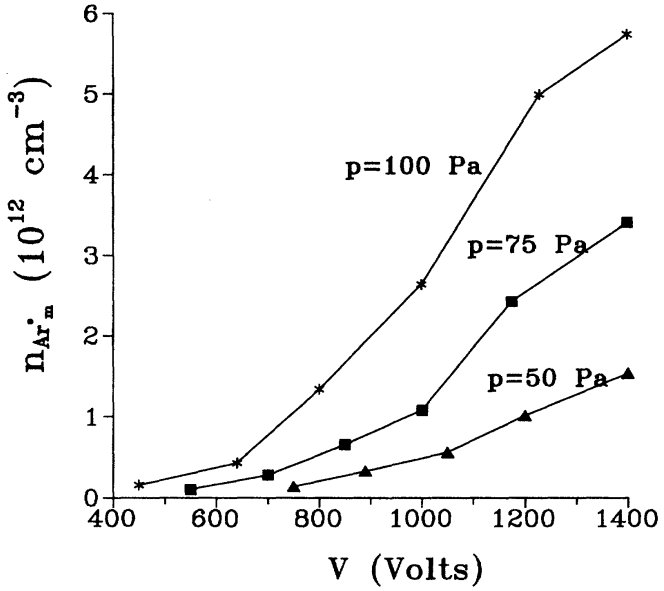

FIG. 7. Ar metastable densities at the maximum of the profile, as a function of voltage at three pressures (Mo in $\mathrm{Ar}$ ).

total amount of ionization in a glow discharge is due not only to Ar ionization, but the ionization of sputtered atoms is not completely negligible. Integrated over the entire discharge region, the relative contributions of $\mathrm{Ar}$ ionization and sputtered atom ionization at these discharge conditions amount to about $99.66 \%$ and $0.34 \%$, respectively. This relative contribution of sputtered atom ionization has not yet been described thoroughly elsewhere. However, the ionization of impurity gas atoms by analogous Penning ionization processes is stated to be important and is the reason why the highest degree of gas purity is desirable when performing physical measurements on electrical discharges in gases [45].

To study the influence of pressure, current, and voltage on the behavior of metastable atoms in the discharge, calculations were performed for a range of voltages, currents, and pressures $\left(V=450-1400 \mathrm{~V}, I=0.2-10 \mathrm{~mA} / \mathrm{cm}^{2}, p\right.$ $=50-100 \mathrm{~Pa}$ ). These are typical discharge conditions used with the VG 9000 glow discharge mass spectrometer (Fisons) for a Mo cathode in Ar.

Figure 6(a) and 6(b) show the Ar metastable density profiles as a function of voltage at $50 \mathrm{~Pa}$ and as a function of pressure at $1000 \mathrm{~V}$, respectively. The metastable density increases with rising voltage and pressure and also shifts closer to the cathode. The reason for this is clear. At high voltages the ion- and atom-impact excitation production processes become increasingly important, resulting in a more clearly defined peak close to the cathode. Moreover, the sheath becomes thinner at higher voltages, which will presumably bring the ion-fast atom and electron-impact peaks closer to each other and in turn closer to the cathode. The influence of the pressure is explained by the increasing diffusion at low pressures, which tends to spread out the density profile. In Fig. 7 the Ar metastable density at the top of the density profile is illustrated as a function of discharge voltage at three pressures. The metastable density clearly increases with increasing pressure and voltage. These results agree rather well with literature data. The increase in metastable density with increasing pressure is also found in Ref. [9]. Other papers $[4,7,14]$ report that the metastable density reaches a maximum at a certain pressure whereafter it decreases again. This pressure value is found to be about 0.7 Torr in Ref. [4] 


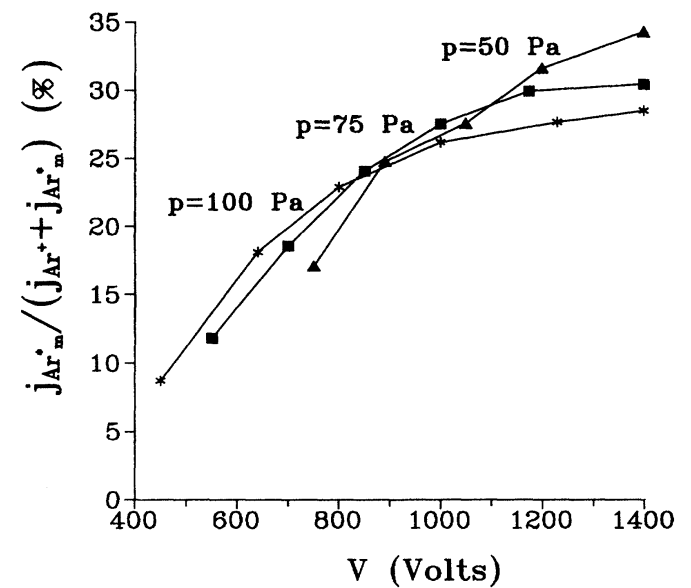

FIG. 8. Contribution of Ar metastable flux to the total flux of Ar ions and metastable atoms at the cathode, as a function of voltage at three pressures (Mo in Ar).

and about 2 Torr in Ref. [14]. It seems that in our model this pressure would be higher than $100 \mathrm{~Pa}(0.75$ Torr), the highest pressure normally used with the VG 9000 mass spectrometer. It is also generally found in the literature that the metastable density increases with current $[4,7,8,11,15]$, although Refs. [7] and [11] illustrate that density saturation occurs at a certain current (i.e., about 6 and $10 \mathrm{~mA}$, respectively).

Figure 8 shows the relative contribution of the Ar metastable flux to the total flux of Ar ions and metastable atoms at the cathode. Since the metastable flux at the cathode varies with pressure to the same extent as the ion flux but increases more rapidly as a function of voltage, the relative contribution of the metastable flux is rather independent of pressure, but it rises with voltage. At $1400 \mathrm{~V}$, it comprises about 30\% of the total flux at the cathode. At still higher voltages, it seems to reach saturation, so that the metastable flux appears never to constitute the major bombarding flux at the cathode. Assuming that the secondary electron emission coefficient of ions and metastable atoms is comparable (see before), this relative metastable flux at the cathode also gives the relative contribution of metastable atoms in secondary electron emission. Hence we conclude that the metastable atoms play an important role in secondary electron emission.

In Fig. 9 the relative contributions of different production and loss processes integrated over the entire discharge are presented, as a function of voltage at $75 \mathrm{~Pa}$ [Fig. 9(a)] and as a function of pressure at $1000 \mathrm{~V}$ [Fig. 9(b)]. The most important production process at the discharge conditions investigated seems to be ion- and atom-impact excitation. This production process increases with voltage and is nearly independent of pressure. Only at voltages of about $500 \mathrm{~V}$ electron-impact excitation appears to be equally important. At still lower voltages ion- and atom-impact excitation will gradually become negligible whereas electron-impact excitation will become the major production process. Concerning the loss processes, diffusion seems to be the dominant one at all voltages and pressures since it tends to balance the high production process near the cathode where electron quenching to the resonant states has a negligible role. The second most important loss process is given by electron quenching.

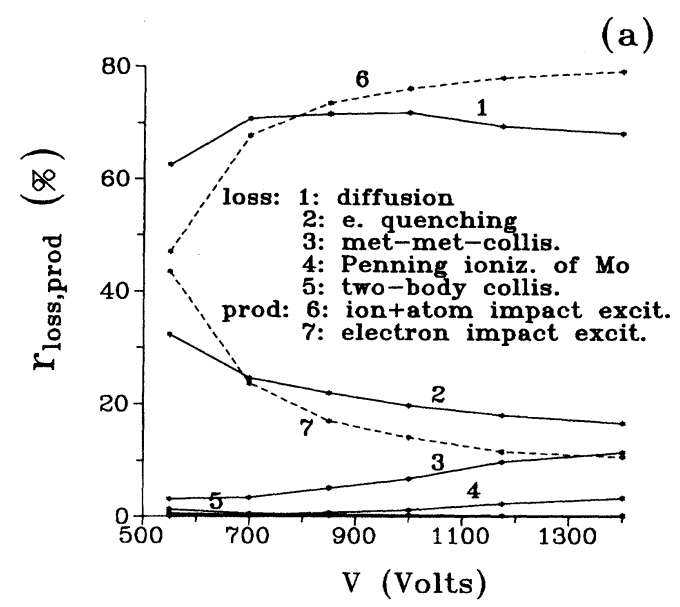

(b)

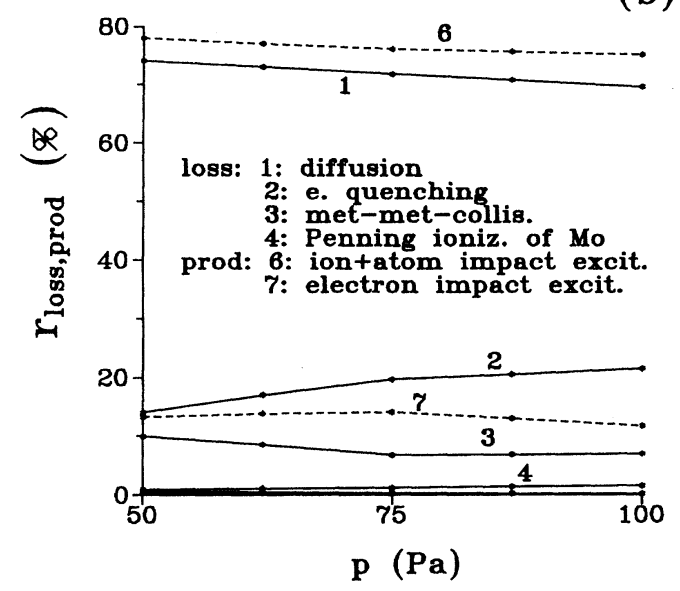

FIG. 9. Contribution of different production and loss processes determining the Ar metastable density, integrated over the entire discharge, (a) as a function of voltage at $75 \mathrm{~Pa}$ and (b) as a function of pressure at $1000 \mathrm{~V}$ (Mo in Ar).

Metastable-atom-metastable-atom collisions also have a clear contribution, whereas Penning ionization of sputtered Mo atoms only becomes non-negligible at high voltages (about $3.5 \%$ at $1400 \mathrm{~V}$ ). These relative contributions of different loss processes remain in the same order of importance at all voltages and pressures investigated. Figure 9(b) shows that pressure has no clear influence on the different loss terms. The voltage seems to have slightly more effect, as is illustrated in Fig. 9(a). Electron quenching is relatively most important at low voltages. At high voltages there is competition from metastable-atom-metastable-atom collisions, a loss process that clearly increases with voltage due to the higher metastable densities. The relative importance of Penning ionization of sputtered Mo atoms also increases with voltage due to the higher sputtered atom densities. Two-body collisions with argon atoms have only a slight contribution at very low voltages. The other processes remain negligible at all discharge conditions investigated.

The influence of pressure and voltage on the relative contribution of metastables to the ionization of $\mathrm{Ar}$ and of sputtered Mo (Penning ionization) was also investigated. Con- 
cerning the ionization of Ar, direct electron-impact ionization of ground-state Ar atoms remains dominant at all voltages and pressures used here, although ion- and especially atomimpact ionization should also be considered at high voltages (i.e., about $6.5 \%$ and $16 \%$ at $1400 \mathrm{~V}$ ). The relative contribution of metastable-atom-metastable-atom collisions to the ionization apparently increases with voltage and decreases with pressure (for example, it constitutes about $0.7 \%$ at 100 $\mathrm{Pa}$ and $450 \mathrm{~V}$ and this value rises to about $8 \%$ at $50 \mathrm{~Pa}$ and $1400 \mathrm{~V})$. The influence of electron-impact ionization from the metastable states is always less than $0.01 \%$ at these discharge conditions.

Concerning the ionization of sputtered Mo, electronimpact ionization remains of minor importance (around 1\%) at all voltages and pressures. The relative contributions of Penning ionization and charge transfer vary more clearly with voltage and pressure since the relative magnitudes of argon metastable atoms and argon ions also vary with these discharge conditions. In general, Penning ionization seems to be more important at low voltages and low pressures. At 50 $\mathrm{Pa}$, it is clearly dominant at all voltages, constituting about $98.7 \%$ at $750 \mathrm{~V}$ and about $91 \%$ at $1400 \mathrm{~V}$. However, at 100 $\mathrm{Pa}$, it is only dominant at low voltages (i.e., about $87.8 \%$ at $450 \mathrm{~V}$ ) whereas it decreases to about $25 \%$ at $1400 \mathrm{~V}$. The increasing importance of charge transfer at higher voltages and pressures was also suggested in Ref. [5]. However, the exact values of the relative contributions of these processes have to be considered with care since they are only reliable if the cross sections of both processes are correct.

Finally, we investigated the influence of pressure and voltage on the relative contribution of the ionization of Ar and Mo in the total amount of ionization. Due to the higher sputtered atom density at higher voltages and pressures, the relative contribution of ionization of Mo increases with pressure and voltage. At voltages of about the threshold values [i.e., about $450 \mathrm{~V}$ (at $100 \mathrm{~Pa}$ ), to about $750 \mathrm{~V}$ (at $50 \mathrm{~Pa}$ )] it constitutes only about $0.05 \%$. However, as voltage increases, the relative contribution of Mo ionization also increases, the increase being even more significant at higher pressures. At $1400 \mathrm{~V}$, this relative contribution is about $1.6 \%$ at $50 \mathrm{~Pa}$ and about $5.5 \%$ at $100 \mathrm{~Pa}$. This indicates that at high voltages and pressures sputtered atoms and ions have a non-negligible influence on the discharge.

\section{CONCLUSION}

A balance equation is constructed to model the behavior of Ar metastable atoms in a dc glow discharge. The discharge conditions range from 50 to $100 \mathrm{~Pa}$ gas pressure and from 500 to $1400 \mathrm{~V}$ discharge voltage, yielding electrical currents from about 1 to about $10 \mathrm{~mA} / \mathrm{cm}^{2}$. Production processes taken into account are electron-, ion-, and atom-impact excitation and radiative recombination. Loss processes include diffusion and deexcitation at the walls, electron-impact excitation and ionization from metastable levels, electron quenching to the nearby resonant levels, metastable-atommetastable-atom collisions, Penning ionization, and two- and three-body collisions with ground-state Ar atoms. The metastable density profile was calculated and compared with the Ar ion and sputtered Mo atom density. A study was made on the relative contribution of different production and loss pro- cesses determining the density of the metastable atoms. Ionand atom-impact excitation were found to be the dominant production processes at $75 \mathrm{~Pa}$ and $850 \mathrm{~V}$. Loss of metastable atoms was primarily due to diffusion, followed by electron quenching, metastable-atom-metastable-atom collisions, and Penning ionization. The other processes were found to be negligible.

The relative role of metastable atoms in the discharge was investigated. Metastable atoms appeared to play a rather important role in the secondary electron emission at the cathode. Their role in the ionization of Ar atoms was only a few percent. They are, however, quite important for the ionization of sputtered Mo atoms: about $70 \%$ of the ionization of Mo was caused by Penning ionization at $75 \mathrm{~Pa}$ and $850 \mathrm{~V}$, the remaining part being due to charge transfer with Ar ions (based on the assumption that both processes have about the same cross section), while electron-impact ionization accounted only for about $1 \%$.

The influence of pressure, voltage, and current on the calculated quantities was examined. The density of metastable atoms increased clearly with voltage and pressure. The relative flux of metastable atoms at the cathode to the total flux of ions and metastable atoms, and hence the relative contribution of metastables in secondary electron emission, was nearly independent of pressure but increased with voltage, reaching values of about $30 \%$ at $1400 \mathrm{~V}$. The pressure and voltage effect on the relative contribution of different production and loss processes was rather small at the discharge conditions investigated; ion- and atom-impact excitation remained the dominant production process except at voltages of $500 \mathrm{~V}$ and less. Also the relative contributions of different loss processes kept the same order of importance at all voltages and pressures, although electron quenching seemed to decrease slightly with voltage whereas metastable-atommetastable-atom collisions and Penning ionization showed a slight increase with voltage. The relative role of metastableatom-metastable-atom collisions in the ionization of Ar increased with increasing voltage and decreasing pressure reaching at maximum a contribution of about $8 \%$. The contribution of electron-impact ionization from the metastable levels was always less than $0.01 \%$. On the other hand, the relative role of metastable atoms in the ionization of Mo, i.e., Penning ionization, was of major importance, especially at the low pressures and voltages. At $100 \mathrm{~Pa}$ charge transfer played a more significant role as the voltage increased. The relative contribution of electron-impact ionization of Mo remained of the order of $1 \%$ at all voltages and pressures. Finally, the contribution of the ionization of Mo atoms on the total amount of ionization in the discharge was investigated and it was found that this contribution was negligible at low voltages and pressures but that it increased with voltage and pressure to about $5.5 \%$ at $100 \mathrm{~Pa}$ and $1400 \mathrm{~V}$.

\section{ACKNOWLEDGMENTS}

A.B. is indebted to the National Science Foundation (NFWO) for financial support. The authors also acknowledge financial support from the Federal Services for Scientific, Technical and Cultural Affairs (DWTC/SSTC) of the Prime Minister's Office through IUAP-III (Conv. 49). 
[1] B. Chapman, Glow Discharge Processes (Wiley, New York, 1980).

[2] W. W. Harrison, in Inorganic Mass Spectrometry, edited by F. Adams, R. Gijbels, and R. Van Grieken (Wiley, New York, 1988), Chap. 3.

[3] Glow Discharge Spectroscopies, edited by R. K. Marcus (Plenum, New York, 1993).

[4] R. L. Smith, D. Serxner, and K. R. Hess, Anal. Chem. 61, 1103 (1989).

[5] M. K. Levy, D. Serxner, A. D. Angstadt, R. L. Smith, and K. R. Hess, Spectrochim. Acta 46B, 253 (1991).

[6] E. W. Eckstein, J. W. Coburn, and E. Kay, Int. J. Mass Spectrom. Ion Phys. 17, 129 (1975).

[7] P. G. Browne and M. H. Dunn, J. Phys. B 6, 1103 (1973).

[8] N. I. Uzelac and F. Leis, Spectrochim. Acta 47B, 877 (1992).

[9] N. P. Ferreira, J. A. Strauss, and H. G. C. Human, Spectrochim. Acta 37B, 273 (1982).

[10] J. A. Strauss, N. P. Ferreira, and H. G. C. Human, Spectrochim. Acta 37B, 947 (1982).

[11] C. M. Ferreira and A. Ricard, J. Appl. Phys. 54, 2261 (1983).

[12] C. M. Ferreira, J. Loureiro, and A. Ricard, J. Appl. Phys. 57, 82 (1985).

[13] T. Kubota, Y. Morisaki, A. Ohsawa, and M. Ohuchi, J. Phys. D 25, 613 (1992).

[14] K. A. Hardy and J. W. Sheldon, J. Appl. Phys. 53, 8532 (1982).

[15] E. A. Den Hartog, D. A. Doughty, and J. E. Lawler, Phys. Rev. A 38, 2471 (1988).

[16] E. A. Den Hartog, T. R. O'Brian, and J. E. Lawler, Phys. Rev. Lett. 62, 1500 (1989).

[17] D. P. Lymberopoulos and D. J. Economou, J. Appl. Phys. 73, 3668 (1993)

[18] A. V. Phelps and J. P. Molnar, Phys. Rev. 89, 1202 (1953).

[19] A. V. Phelps, Phys. Rev. 99, 1307 (1955).

[20] E. Ellis and N. D. Twiddy, J. Phys. B 2, 1366 (1969).

[21] G. H. Copley and C. S. Lee, Can. J. Phys. 53, 1705 (1975).

[22] K. Tachibana, Phys. Rev. A 34, 1007 (1986).

[23] J. H. Kolts and D. W. Setser, J. Chem. Phys. 68, 4848 (1978).
[24] A. Bogaerts, R. Gijbels, and W. J. Goedheer, J. Appl. Phys. 78, 2233 (1995).

[25] J. A. Valles-Abarca and A. Gras-Marti, J. Appl. Phys. 55, 1370 (1984).

[26] M. van Straaten, A. Vertes, and R. Gijbels, Spectrochim. Acta 46B, 283 (1991).

[27] Y. Yamamura, N. Matsunami, and N. Itoh, Radiat. Eff. 71, 65 (1983).

[28] A. Bogaerts, M. van Straaten, and R. Gijbels, Spectrochim. Acta 50B, 179 (1995).

[29] A. Bogaerts, M. van Straaten, and R. Gijbels, J. Appl. Phys. 77, 1868 (1995).

[30] N. J. Mason and W. R. Newell, J. Phys. B 20, 1357 (1987).

[31] A. Bogaerts and R. Gijbels, J. Appl. Phys. 78, 11 (1995).

[32] A. V. Phelps and B. M. Jelenkovic, Phys. Rev. A 38, 2975 (1988).

[33] M. A. Biondi, Phys. Rev. 129, 1181 (1963).

[34] H. A. Hyman, Phys. Rev. A 20, 855 (1979).

[35] H. A. Hyman, Phys. Rev. A 18, 441 (1978).

[36] L. A. Riseberg, W. F. Parks, and L. D. Schearer, Phys. Rev. A 8, 1962 (1973).

[37] S. Inaba, T. Goto, and S. Hattori, J. Phys. Soc. Jpn. 52, 1164 (1983).

[38] D. U. von Rosenberg, Methods for the Numerical Solution of Partial Differential Equations (Elsevier, New York, 1969), p. 113.

[39] W. Vieth and J. C. Huneke, Spectrochim. Acta 45B, 941 (1990).

[40] L. Vriens, Phys. Lett. 8, 260 (1964).

[41] E. B. M. Steers and R. J. Fielding, J. Anal. At. Spectrom. 2, 239 (1987).

[42] P. Baltayan, J. C. Pebay-Peyroula, and N. Sadeghi, J. Phys. B 18, 3618 (1985).

[43] P. Baltayan, J. C. Pebay-Peyroula, and N. Sadeghi, J. Phys. B 19, 2695 (1986).

[44] E. B. M. Steers and F. Leis, J. Anal. At. Spectrom. 4, 199 (1989).

[45] F. Llewellyn-Jones, Ionization and Breakdown in Gases (Science Paperbacks, Norwich, 1966), p. 36. 